\title{
Creación de Redes Interorganizativas en un Cluster Territorial
}

\author{
Josep Capó-Vicedo, Manuel Expósito-Langa y José V. Tomás-Miquel \\ Universidad Politécnica de Valencia, Departamento de Organización de Empresas, Escuela \\ Politécnica Superior de Alcoy, Plaza Ferrándiz y Carbonell, 03801 Alcoy, Alicante-España \\ (e-mail: pepcapo@doe.upv.es)
}

\begin{abstract}
Resumen
El objetivo del presente trabajo es proponer una metodología para la creación de redes interorganizativas, en el caso concreto de PYMEs localizadas en clusters territoriales. Para ello se realizará una breve revisión de alguna de las aportaciones más significativas en la literatura existente sobre las agrupaciones territoriales de empresas o clusters, así como sobre las redes sociales y las empresas virtuales. Del análisis realizado se concluye que la metodología propuesta debe centrarse en el análisis de las relaciones con otras empresas y agentes externos e internos al territorio en el que se ubican para la obtención de ventajas competitivas. Esta metodología se plantea como complementaria a las ya "tradicionales" de análisis estructural de un sector.
\end{abstract}

Palabras clave: clusters territorial, PYME, redes interorganizativas, estrategia, competitividad

\section{Proposal for a Methodology for Creating Interorganizational Networks in Territorial Clusters}

\begin{abstract}
The aim of this paper is to propose a methodology for creating interorganizational networks is proposed for the case of SMEs located in territorial clusters. To achieve this objective, a brief revision of the existing literature related to territorial concentrations of firms (clusters), social networks and virtual organizations is done. From this analysis, it is concluded that the proposed methodology must be centred on the analysis of relationships with other companies and external and internal agents of the territory in which they are located, with regards to trying to obtain competitive advantages. We consider this kind of analysis to be complementary to traditional structural analyses of a sector.
\end{abstract}

Keywords: territorial clusters, SME, interorganizational networks, strategy, competitivity 


\section{INTRODUCCIÓN}

En los últimos años los investigadores han mostrado un creciente interés en la estructura y la fortaleza de las relaciones en los sistemas sociales, surgiendo un marco teórico común para entender este fenómeno que descansa en el concepto del Capital Social.

Por otro lado, la proximidad geográfica, por lo que representa en cuanto a la frecuencia e intensidad de las relaciones, condiciona, si no determina, una caracterización específica del capital social. En concreto, los modelos territoriales como el distrito industrial o el cluster analizan el impacto de la proximidad geográfica de las empresas y las instituciones. Estos modelos sugieren implicaciones importantes para la creación y desarrollo del capital social de los actores participantes.

Los teóricos del capital social están de acuerdo en que, los actores mejor conectados en la red poseen una ventaja competitiva sobre los pobremente conectados. Sin embargo, el desacuerdo aparece respecto a que significa estar mejor conectados. El argumento de Coleman (1988) y Uzzi (1997) de las redes cerradas, de fuertes vínculos, sugiere la supremacía de las redes densas. Por el contrario, Burt (1997), prescribe que las empresas han de estar conectadas en redes dispersas de vínculos débiles (Granovetter, 1985) de socios desconectados. Un número significativo de autores han propuesto una aproximación conciliadora de las dos perspectivas (Rowley et al., 2000; Dyer y Nobeoka, 2000, entre otros).

El análisis de redes proporciona numerosas herramientas para dibujar la estructura de las relaciones o vínculos interorganizacionales, basándose en el reconocimiento de que la estructura de la red impone y al mismo tiempo modela las acciones de las empresas (Granovetter, 1985; Burt, 1997; Nohria, 1992). No obstante, aunque esta perspectiva de redes acentúa la importancia de las interdependencias, entre múltiples empresas, y cómo las relaciones interorganizacionales pueden ser una importante fuente de ventaja competitiva, la operativa para crear estas redes está todavía poco estudiada. Este trabajo intenta llenar este vacío, proponiendo una metodología que pretende dar una primera aproximación para el caso particular de empresas concentradas en localizaciones geográficas concretas, formando clusters.

Para alcanzar este objetivo, previamente se realizará un repaso a las principales teorías que son la base de la metodología propuesta. En concreto se estudiarán las principales características de los clusters, de las redes sociales, así como las de las organizaciones o empresas virtuales. A partir de este estudio se determinarán los principales puntos que debería cubrir la metodología que se propone en el presente trabajo, pasando finalmente a describir la misma.

\section{ANTECEDENTES}

Hoy en día el mundo se encuentra globalizado, donde el uso de las tecnologías de la información y la comunicación (TIC), y de sistemas logísticos avanzados, permite el establecimiento de relaciones con empresas de cualquier parte del mundo. No obstante, no deja de ser cierto que, para el establecimiento de alianzas exitosas entre empresas, es muy beneficiosa la existencia de los llamados "ecosistemas de negocios" (Camarinha-Matos, 2002), es decir entornos que favorezcan este tipo de configuraciones en red, a través de la existencia de prácticas y culturas empresariales similares, de confianza entre las empresas, y de un sentimiento de comunidad y estabilidad. Estos ecosistemas o entornos favorecedores se pueden encontrar, por ejemplo, en forma de redes de empresas especializadas, localizadas en concentraciones territoriales concretas en gran número de países avanzados (Italia, España, etc.), formando clusters.

\section{Clusters}

Fue Porter (1990), el que empezó a hablar de los clusters, poniendo de manifiesto que las razones del éxito competitivo internacional estaban asociadas a dos causas: a) la concentración geográfica de agentes económicos especializados; y b) la presencia de los adecuados factores determinantes de la competitividad territorial, que han permitido a las empresas de estos clusters especializados alcanzar elevados niveles de éxito. 
Este éxito de las redes de empresas especializadas, localizadas en concentraciones territoriales concretas en gran número de países avanzados, ha aumentado el interés por el estudio de estos fenómenos de concentración productiva geográfica durante las dos últimas décadas, tanto por parte del mundo académico, como por parte de los responsables de la elaboración de las políticas industriales y de desarrollo territorial.

Durante la pasada década, los clusters han sido reconocidos como escenarios en los que se dan condiciones muy favorables ya sea para el estímulo de la productividad y de la innovación en las empresas que los integran, ya sea para la formación de los recursos humanos o para la creación de nuevos negocios. De hecho, durante las últimas dos décadas los investigadores han mostrado una creciente atención por las empresas localizadas en áreas geográficas limitadas. Como resultado de este interés se pueden encontrar una gran variedad de conceptos explicativos del fenómeno: La Especialización Flexible (Piore y Sabel, 1984); El sistema de producción (Storper y Harrison, 1991); el Cluster regional (Porter, 1990) el sistema nacional de innovación (Lundvall, 1992) o el hot spot (Pouder y St. John, 1996). Sin embargo como señalan Storper y Harrison (1991), el gran número de conceptos y aproximaciones produce confusión y dificulta la comprensión del fenómeno.

\section{Redes Sociales}

En todos estos estudios ha quedado patente la importancia que plantea la existencia de una red estructural de relaciones entre los agentes económicos de un cluster, la cual es generadora de economías externas de valor para la mayoría de ellos. Lo anterior queda refrendado si se tiene en cuenta que, a priori, la escasez de recursos a la que estructuralmente se enfrentan las PYMEs, dificulta que éstas, por sí mismas, estén preparadas para abordar los procesos de cambio estratégico, imprescindibles en los actuales y turbulentos escenarios de la nueva economía globalizada. Sin embargo, se da la paradoja de que muchos de los productos manufacturados de calidad, así como gran número de servicios especializados avanzados, son producidos con éxito dentro de clusters, por agrupaciones de PYMEs concentradas geográficamente, operando en red.

La perspectiva social o de redes, nace como contraste a una visión atomística, que asume que la empresa actúa sola y se entiende como la perspectiva del arraigo (embeddedness). Esta perspectiva subraya el papel de las redes sociales para explicar las acciones económicas de la empresa (Granovetter, 1985). Dentro de esta corriente teórica algunos autores han puesto el énfasis en el conjunto de recursos arraigados en las redes sociales (Burt, 1997), mientras que otros autores han extendido el estudio más allá de los recursos de las relaciones sociales incluyendo también las normas y valores asociados con ellas (Coleman, 1988; Putman, 1995).

El trabajo clásico de Granovetter (1985), sirve como punto de referencia para la investigación actual sobre el arraigo. Su argumento del arraigo sugiere que la conducta y las instituciones económicas están afectadas por relaciones sociales continuas y que éstas no pueden entenderse de forma separada o independiente. La aproximación de Granovetter acentúa el papel que pueden desempeñar las relaciones sociales en la generación de la confianza y en limitar o desalentar la desconfianza entre las partes (Oinas, 1998).

La confianza es característica explícita y primaria de las relaciones arraigadas. La confianza expresa la creencia de que el socio del intercambio no actuará en su propio interés en los aspectos que afectan al otro. Adicionalmente el socio no actúa con un riesgo calculado sino su actuación tiene un carácter heurístico. La información intercambiada en las relaciones arraigadas, tiene un carácter más de propiedad y tácito que la información que de precios y cantidades que se comercia en los vínculos a distancia. Los acuerdos de resolución conjunta de problemas, mejora las respuestas de la organización al reducir la producción de errores y el número ciclos de desarrollo. Los acuerdos de resolución conjunta de problemas son mecanismos de voz y reemplazan la respuesta simplista de salir o quedarse del mercado y enriquece la red. Al trabajar sobre los problemas estos mecanismos promueven el aprendizaje y la innovación.

Dado que uno de los factores clave será, precisamente, el establecimiento y potenciación de estas redes sociales, en el siguiente apartado se entrará en detalle en una tipología de redes 
interorganizativas que, por sus características, se ajusta perfectamente a las necesidades vistas en este apartado de las empresas que forman un cluster, para garantizar su competitividad.

\section{Empresa Virtual}

La Empresa Virtual puede considerarse como una evolución particular de la organización en red, en la cual los participantes intentan establecer una forma de gestionarse y organizarse más "democrática", en cuanto a la gestión de los flujos de información, bienes, decisiones y control.

Actualmente, se dan muchos casos en los que los procesos productivos ya no se llevan a cabo por una única empresa, sino por organizaciones cooperativas en red, donde cada empresa es un nodo que añade valor al proceso global. Según Martínez et al. (2001), el principal objetivo de este tipo de organizaciones es permitir a un grupo de empresas desarrollar de forma rápida, un entorno de trabajo común, gestionando los recursos de cada una de ellas, de forma que se alcancen una serie de objetivos comunes.

En este contexto aparece la Empresa Virtual, como respuesta estructural basada en la flexibilidad, la adaptabilidad, la oportunidad y la optimización de la estructura de costes, de tal forma que el conjunto formado sea capaz de responder eficaz y rentablemente a las necesidades de los clientes (Cuesta, 1998).

La revisión de la literatura aporta muchas definiciones de Empresa Virtual (EV). Una de las primeras fue presentada por The Economist en un trabajo del 6 de febrero de 1993: "Red temporal de empresas que se unen para explotar una oportunidad específica de mercado apoyada en las capacidades tecnológicas de las empresas que forman la red".

Todas las definiciones coinciden en definirlas como redes de empresas colaboradoras, las cuales actúan como nodos de la misma, aportando cada una lo que sabe hacer mejor que ninguna otra (Core Business). Todas ellas operan de cara al cliente como si de una única empresa se tratara. El resultado será la consecución de una estructura de costes óptimos.

A continuación se analizan brevemente las etapas del ciclo de vida de una Empresa Virtual, así como los principales requerimientos en cada una de ellas, los cuales serán los que pretende cubrir la metodología propuesta en este trabajo.

Según Camarinha-Matos y Afsarmanesh (1999), las fases del ciclo de vida de una Empresa Virtual (EV) son cuatro; creación, operación, evolución y disolución. En este trabajo se propone añadir una fase adicional, la de gestación, la cual será clave en la metodología propuesta, con lo cual el ciclo de vida de la EV quedaría de la siguiente manera:

Gestación: En esta primera fase se define el ámbito de actuación de la EV y se establece el marco estratégico en el que se moverá la misma. Para ello deberá identificarse en primer lugar una oportunidad de mercado específica, definiéndose posteriormente las características de la EV a crear para responder a la misma. El desarrollo de esta fase es de gran importancia, ya que condicionará las siguientes fases del ciclo de vida de la EV. Una incorrecta definición de estas características puede implicar problemas en su funcionamiento, obligando a reconfiguraciones o cambios posteriores, con toda la problemática que esto implica.

Creación: Esta es la fase en la cual la EV se crea o construye. Una vez se han establecido las características que debe tener la EV se inicia la creación de la misma. Cada oportunidad de negocio a afrontar implicará la creación de una EV concreta, en la cual se integrarán distintas empresas en función de sus características particulares, y de lo establecido en la fase anterior.

Operación: En esta fase se realizan los procesos de negocio, buscando conseguir los objetivos comunes. En ella se necesitan mecanismos de intercambio seguro de datos, de intercambio de información, gestión de órdenes, planificación y programación, gestión de distribución de tareas, coordinación de tareas, etc. Una vez establecida la EV empieza esta fase operacional, en la cual se dan distintos niveles de interacción entre sus miembros. 
Evolución: La evolución es una fase que se presenta durante el funcionamiento de una EV, cuando es necesario añadir o reemplazar algún miembro. Esto puede deberse a algún suceso inesperado como la incapacidad temporal de alguno de ellos, a un aumento de la carga de trabajo, etc.

Disolución: En esta última fase la EV finaliza sus procesos de negocio y debe "desmantelarse". Esto puede darse por dos motivos: la consecución satisfactoria de sus objetivos, o la decisión de alguno de sus miembros de parar la ejecución de la EV.

Una vez vistas brevemente las principales características de las empresas virtuales, se presenta la propuesta de una metodología que permita crear organizaciones de este tipo en el seno de clusters económico-productivos. Para ello, lo más importante será la identificación de las oportunidades de negocio que pueden motivar la aparición de una Empresa Virtual, así como la determinación de los componentes del cluster más apropiados para formar la misma y hacer frente a la oportunidad de negocio correspondiente, es decir la determinación de los parámetros necesarios para las dos primeras etapas del ciclo de vida de una Empresa Virtual, la gestación y la creación.

\section{METODOLOGÍA PROPUESTA}

A partir de los conceptos explicados, se propone en este trabajo una metodología capaz de identificar oportunidades de mercado en un cluster, así como los agentes del mismo más apropiados para constituir una red interorganizativa, del tipo de una empresa u organización virtual, para afrontarla con garantías de éxito.

\section{Identificación de las oportunidades de negocio}

Para alcanzar el objetivo del presente trabajo, el modelo genérico de Porter sobre la Cadena de Valor no es suficiente. Este modelo, al diferenciar las actividades a realizar en la producción de cualquier bien o servicio en actividades principales y actividades secundarias o auxiliares, es insuficiente para aplicar un análisis estratégico a escala de un cluster. Se precisa de un mayor nivel de detalle en el análisis para diagnosticar cuál o cuáles son los factores clave de éxito que específicamente deben llevar a las empresas a adoptar decisiones concretas en relación con el resto de agentes económicos con los que está interrelacionado: clientes, proveedores, empresas conexas y relacionadas, instituciones públicas y/o privadas, etc.

Por otra parte, no se debe olvidar que el modelo de la Cadena de Valor fue definido inicialmente por Porter para una empresa concreta y esto supone una limitación adicional para ser aplicado en el estudio analítico en el caso de clusters de empresas. Precisamente, la interrelación entre las fases productivas de un sector industrial, ejercidas por empresas diferentes, pero geográficamente próximas, es lo que permite plantear el concepto de Cadena de Valor extendida a todo el proceso productivo que tiene lugar en un determinado territorio, sobre la base de su relación en términos de producto/mercado. Ello da lugar a un nuevo concepto que puede facilitar el proceso del análisis estratégico pretendido: el Marco de la Cadena de Valor de un cluster (Masiá et al., 2004).

A escala de un cluster definido en términos de producto/mercado se pueden identificar todas y cada una de las fases productivas concretas que se ejecutan, para transformar los inputs iniciales en bienes y servicios útiles para los usuarios finales de los mercados objetivo elegidos. Al mismo tiempo, al centrarse en una fase productiva, se pueden identificar también con precisión las tecnologías específicas que se usan o se pueden utilizar para ejecutarla de la forma más eficaz y eficiente.

En el diseño inicial del Marco, al considerar las actividades así definidas, no se tiene en cuenta cual o cuáles empresas las realizan, y si todas ellas están o no dentro del territorio considerado. Se trata de dibujar el proceso "habitual" o "tradicional" en base al cual se viene produciendo hasta hoy un producto o servicio en el territorio considerado, independientemente de las empresas concretas que lo ejecuten.

Una fase, etapa o eslabón de la cadena de valor queda identificada sin ambigüedades a partir de estas dos características; tiene un suceso inicial y un suceso final perfectamente definidos (hitos);

Información Tecnológica - Vol. 18 Nº 5 - 2007 
genera valor, aunque en ocasiones este puede ser muy bajo o nulo, en términos de la apreciación del consumidor final, y tiene unos costes asociados a las actividades desarrolladas, correspondientes al coste económico de los recursos consumidos para la ejecución de las mismas (materiales, horas de trabajo, energía, información, gestión, etc.)

Se puede así, a partir de la anterior información, diseñar el Marco específico de la Cadena de Valor de un cluster, dibujando un gráfico en forma de diagrama de flujo con bloques o cajas que representen los eslabones. En este diagrama, cada cuadro o bloque debe corresponder a una fase perfectamente delimitada que corresponda a una actividad que genere valor y tenga unos costes asociados, en función de los recursos consumidos para ejecutarla. Este nivel de detalle permite un análisis crítico de cada actividad concreta realizada, relacionando precisamente el valor generado para el mercado de la misma con sus costes asociados específicos.

Por otra parte, hay que tener en cuenta que cada una de estas fases puede realizarse por agentes internos o por agentes externos al territorio considerado, poniendo de manifiesto las oportunidades de introducir valor dentro del mismo, en aquellos casos en que la relación valor/coste sea muy importante y se esté ejecutando por empresas localizadas en el exterior del territorio, ajenas, por lo tanto, al cluster.

Así, una vez dibujado el Marco de la Cadena de valor de un cluster, se debe actuar a dos niveles; análisis individual de cada uno de los eslabones de la cadena y análisis del posicionamiento de una fase concreta en la secuencia de eslabones y posibilidades de cambio y/o de diferente relación.

A partir de aquí se podrán identificar las oportunidades de negocio que constituyan los factores clave de éxito; es decir, el conjunto de recursos y capacidades que debe poseer una empresa para utilizarlos en acciones generadoras de valor o reductoras de costes por encima de sus competidores. La identificación de estos factores deberá hacerse, principalmente, a través de un proceso creativo donde la información relevante en cantidad y calidad, y la experiencia de los agentes responsables de la toma de decisiones en el campo empresarial, permita generar distintas alternativas estratégicas de futuro y les permita intuir cuál o cuáles de ellas tienen mayor potencial generador de valor.

La información y el conocimiento generados tanto en el proceso de análisis de la estructura de los clusters en términos de producto/mercado, como en el proceso de el análisis crítico del Marco de la Cadena de Valor, deben proporcionar la generación de alternativas estratégicas suficientes para que los dirigentes empresariales e institucionales del cluster decidan cuáles son los factores clave de éxito que hay que abordar para iniciar procesos estratégicos de cambio, estableciéndose organizaciones virtuales que aborden la oportunidad de negocio asociada a cada factor de éxito.

\section{Identificación de los agentes del cluster que deben formar la EV}

Ya se ha comentado que los factores clave de éxito deben acabar en procesos clave, en los cuales las empresas actúen generando valor por encima de la competencia. Aquí vuelve a aparecer la problemática de la necesidad acción de las empresas en red,... ¿qué agentes del cluster deben asociarse para formar una organización virtual?, ¿cómo conseguir que estos actúen coordinadamente?

Si, como se ha argumentado al analizar el modelo de los cluster, la cantidad y, sobre todo, la calidad de las relaciones existentes entre los principales agentes económicos es la base del éxito del mismo en términos de competitividad, es necesario analizar dicha red estructural de relaciones, con el fin de conocer cuántas y cuáles son éstas, y en qué sentido deben orientarse en los futuros procesos de cambio.

Para ello, se propone la utilización de la tabla "Matriz Estructural de Relaciones" (Masiá et al., 2004), la cual es una matriz cuadrada o tabla de doble entrada que va a servir para relacionar entre sí a los principales agentes económicos que están instalados en un determinado territorio: empresas e instituciones. Los elementos de la tabla son los diferentes tipos de agentes económicos del cluster que previamente se han identificado; Instituciones: Horizontales - territoriales y Verticales sectoriales y Empresas: Líderes del cluster, Proveedores, empresas Relacionadas y Clientes. 
Al colocar la lista de los distintos agentes en la primera fila y la primera columna de la MER, el sentido que adquiere cada una de sus celdas es el contenido de la relación que mantiene el Agente $X$ con el Agente $Y$. La relación entre estos dos agentes $X<>Y$ puede adoptar diferentes actitudes: de competencia, de cooperación y de indiferencia. Puede ser formal o informal, y puede generar valor o destruirlo para el conjunto del cluster. Lo generará con una actitud proactiva de cooperación e, incluso de competencia basada en la calidad y el servicio, lo destruirá con una actitud negativa de competir sólo en precios.

Al haber identificado previamente a los principales agentes económicos del cluster y al haber generado una lista de alternativas estratégicas sobre la base de los factores clave de éxito que se intuyen deben ser los soportes del nivel competitivo del cluster, la MER permite establecer el escenario actual de cómo se desarrollan las relaciones entre los agentes económicos más significativos, relación estudiada bajo la luz de cada uno de los factores identificados (oportunidades de negocio). A partir de aquí se analiza cuál es la situación inicial de la red de relaciones contractuales existentes entre los principales agentes del cluster, estudiándolas bajo el punto de mira de los factores clave de éxito determinados según el punto anterior, y, a partir de la misma se identifican los agentes que deben participar para afrontar la oportunidad de mercado, constituyendo la Empresa Virtual.

Con esto se tendría, pues, una primera aproximación a una metodología capaz de cubrir las dos primeras etapas del ciclo de vida de una EV (etapas clave para su éxito), en el caso particular de las empresas y organizaciones que forman clusters económico-productivos.

\section{CONCLUSIONES}

Se ha realizado en este artículo, una propuesta de metodología para la creación y potenciación de redes interorganizativas dentro de un cluster. Para ello, se han analizado en primer lugar las principales características de las redes interorganizativas, así como de los clusters, para pasar posteriormente a definir las principales etapas que deberían llevarse a cabo para poder determinar claramente las oportunidades de negocio que pueden motivar la creación de estas redes, así como los agentes más apropiados del cluster para hacerlo.

A partir de estos requerimientos iniciales se ha propuesto una metodología la cual, aunque sencilla, puede ser de utilidad para las empresas localizadas en clusters, en el sentido de que les puede servir de guía para la realización de un análisis estratégico conducente a la detección de oportunidades concretas en su entorno específico, así como para el establecimiento de las estructuras organizativas más adecuadas para hacerles frente, a partir de la colaboración y la cooperación entre ellas.

Al mismo tiempo, este trabajo puede suponer un primer paso para futuros trabajos de investigación, en los cuales se realice la aplicación empírica de la misma y su posterior validación en diferentes clusters y sectores. Como líneas de investigación complementarias quedarían el análisis de la complejidad de la integración de organizaciones con culturas empresariales diversas o el propio proceso de innovación dentro de los clusters, y la integración del enfoque de sistemas logísticos.

\section{REFERENCIAS}

Burt, R.S.; Contingent value of the social capital. Administrative Science Quarterly: 42, 339-365 (1997).

Camarinha-Matos, L.M.; Collaborative Business Ecosystems and Virtual Enterprises. Kluwer Academic Publishers, Boston, USA: 13-15 (2002).

Camarinha-Matos, L.M. y H. Afsarmanesh; The Virtual Enterprise Concept. Kluwer Academic Publishers, Boston, USA: 3-14 (1999).

Coleman, J.S.; Social capital in the creation of human capital, American Journal of Sociology: 94, 95120 (1988). 
Cuesta Fernández, F.; La Empresa Virtual. La estructura Cosmos. Soluciones e Instrumentos de transformación en la empresa. Mc Graw Hill. Barcelona, España: 20-23 (1998).

Dyer, J.H. y K. Nobeoka; Creating and managing a high performance knowledge sharing network: The case of Toyota, Strategic Management Journal: 21, 345-367 (2000).

Granovetter, M.; Economic Action and Social Structure: The problem of Embeddedness, American Journal of Sociology: 91, 481-510 (1985).

Lundvall, B.A.; National Systems of Innovation: Towards a Theory of Innovation and Interactive Learning. Pinter Publishers, Londres:116-128 (1992).

Martinez, M.T.; P. Fouletier, K.H. Park y J. Favrel; Virtual enterprise: organisation, evolution and control. International Journal of Production Economics: 74(1), 225-238 (2001).

Masiá, E., J. Albors, E. Golf y J. Capó; Identifying key technology success factors in an industry by the application of an analysis model -STRELNET- to the links of the productive value chain among the main economic agents of a microcluster. Paper IAMOT 2004. Washington D.C. USA (2004).

Nohria, N.; Introduction: is a network perspective a useful way to studying organizations? en Networks and Organizations: Structure, Form and Action by Nohria, N. y Eccles, R.G. (Eds.), Harvard Business School Press, Boston, USA: 1-22 (1992).

Oinas, P.; The Embedded Firm?. Prelude for a revived geography of enterprise. Helsinki School of Economics and Business Administration, Helsinki, Finland (1998).

Piore, M.J. y C. Sabel; The Second Divide. Possibilities for Prosperity. Basic Books Inc. Nueva York, USA (1984).

Porter, M.E.; The Competitive Advantage of the Nations. The Free Press. Nueva York, USA (1990).

Pouder, R. y C.H. St. John; Hot spots and blind Spots: Geographic clusters of firms and innovation. Academy of Management Review: 21, 119-225 (1996).

Putman, R.D.; Bowling alone: America's declining social capital. Journal of Democracy: 6, 65-78 (1995).

Rowley, T., D. Behrens y D. Krackhardt; Redundant governance structures: an analysis of structural and relational embeddedness in the steel and semiconductor industries, Strategic Management Journal: 21, 369-86 (2000).

Storper, M. y B. Harrison; Flexibility, hierarchy and regional development: the changing structure of industrial production systems and their forms of governance in 1990's, Research Policy: 20, 407-422 (1991).

Uzzi, B.; Social structure and competition in interfirm networks: the paradox of embeddedness, Administrative Science Quarterly: 42, 35-67 (1997). 